\title{
Applications and Prospects of Sensor in the field of Sports
}

\author{
Jin-wei WANG \\ College of Physical Education \\ Anhui Polytechnic University, \\ Wuhu, Anhui, P.R. China \\ wangjinwei0402@126.com;
}

\begin{abstract}
Sensor technology is rapidly developing and has penetrated into the field of sports. Give the specific application of sensor technology in some field of sports, working principle and characteristics of the various sensors. Point out sensor in sports engineering practices will be increasingly widespread and deepening.
\end{abstract}

Keywords-sensor technology; sports field; applications; prospects

\section{I . INTRODUCTION}

With the development of human society, sports career has also been rapidly developed, which purpose is development of physical strength, enhance physical fitness, and has become one of the important means of international exchanges. Its size and level of development is an important symbol to measure development and progress of a country and social. Therefore, all countries are in great efforts to develop the country's sports career.

At the same time, the science and technology rapid development of science and technology elements have infiltrated into the field of sports and even everywhere. Sports instruments and equipment is an important part of the sport, can help athletes and coaches to get a more comprehensive and accurate sports data. Especially in the large-scale sporting events of the last few years or decades, sports technology based on information, computer ,communication technology add infinite charm to sports, so that people not only enjoy the sport, but also enjoy the information age technology sports vivid, interesting and exciting. To some extent, the competition in the sports arena is not only a contest between athletes, scientists in the field of sports, but also is a technology contest .It has become the logo of a sports power.

In a variety of sports information acquisition system, the sensor is a front-end component to obtain information and in the key components. Its performance directly affects the system performance merits. In order to obtain accurately the time of the game, throwing distance and training intensity of athletes, the sensor technology plays an important role. Sensor technology is continuously applied to the match referee, sports training equipment, the public fitness equipment, Based on the implementation of the development strategy of "science and technology body, sensor technology will be an increasingly wide range of applications in the field of sports and will also be deepening.

\section{II . SENSOR IN THE MATCH REFEREE SYSTEM}

\section{A. Sensor of Distance Measuring in Field Events}

As we all know, the distance is the main basis of the modern field events. Precise determination of the distance of jumping and throwing is one of the most critical of referee.

Performance measurement has been more difficult to the referee in the field events. The traditional method of measurement is measured with measuring tape, steel rule, such as the scene manually. Until 1972, achievements test for the first time to use a state-of-the-art electronic timer and laser rangefinder at the twentieth Munich Olympics in the Federal Republic of Germany, it declared of the end of the era of the stopwatch, measuring tape. These devices were quickly and accurately and automatic to speed up the development of performance measurement techniques in the field events.

There is infrared, ultrasonic, laser technology to measure the distance. They work the same basic principles, the light (sound waves) spreads in the air, is reflected from an object, calculates the distance according to the transmitting and receive light (sound waves).

When ultrasonic wave spread in the air, its speed affect by temperature, humidity, dust, atmospheric pressure, air flow and others, including temperature maximum, therefore, ultrasonic distance measurement accuracy is lower, generally $0.5 \%$ of range. Currently, ultrasound can only meet the test of the application of the non-professional, has been rarely used in competitive sports competitions.

Because infrared refractive index very small when it across other substances, so the long-distance range finder will consider infrared .Its advantage is cheap, easy, security, poor low accuracy and directionality.

The laser beam emitted to the target forms a bright spot on the object surface. The spot imaged onto the photosensitive surface by the imaging objective, the photosensitive receiver to produce the electrical signal corresponding spot positions. The advantage of laser ranging is accurate. The Bosch laser range finder's accuracy is about $1.5 \mathrm{~mm}$. The disadvantage is the need to pay attention to human security, and the difficulty of making, a higher cost.

Since the principle of the propagation of light with respect to the sound, the propagation of light is more stable. Laser ranging is faster than the speed of ultrasonic ranging, more accurate, higher accuracy and stability. Also laser rangefinder is called Throwers Mirror. 
The laser sensor witnessed the field events of major sports events such as shot put, discus, javelin, hammer, measuring the competition results for these contestants, with its high-precision, high stability, good performance, witnessed the birth of competition medals.

\section{B. Track Events Timing Sensor}

Track events decide the athletes ranking by the time. It is timing from the first stopwatch to later fully automatic endpoint camera.

The image acquisition core of the automatic camera of end point is an image sensor, which is gathered together by the array of photosensitive elements and the charge transfer device. The most commonly used photosensitive member is CCD and CMOS.

CCD sensor uses the MOS capacitor made of a silicon semiconductor material as a basic unit. Each photosensitive unit generates an electric charge when its surface is exposed to light. CCD can achieve the transfer of the photo-charge , and convert the charge signal to a voltage or current signal output by applying a certain timing's the high and low on the electrode.

CCD is widely used in television camera of the dim light due to its low noise since 1970 to achieve image acquisition, storage, transmission, processing and reproduction.

The CMOS image sensor is composed by the number of photosensitive cells. Depending on the photosensitive pixel structure it can be divided into the grating type and photodiode

type.

According to whether the sensitive unit has a zoom function it can be divided into a passive pixel image sensors (PPS) and an active pixel image sensors (APS). Active pixel sensor combines the advantages of the CCD and the X-Y addressing, will be allowed to reach 5 um pixel pitch BY $0.25 \mathrm{um}$ process, is accessible high-grade CCD level, suitable for high-performance scientific imaging and low light imaging.

Currently automatic image timing system results by hundredths of a second accurate to a thousandth of a second in international competitions

\section{Boxing Match Sensor}

Boxing match, in order to judge whether felling athlete's shoulders touch the ground, the referee needs to lie on the ground intense observation of the athlete's every move.

Today, the pressure sensor can displayed their prowess, can be installed on both sides athlete's body to collect pressure signal of athlete's shoulders with the ground at the race like taekwondo, fencing competitions using electronic protective gear. When hit a brace effective part the force sensor output electrical signal to the processing circuit, using a wireless network to transfer the data to the receiving end of the referee seats, this judgment system is more accurate efficient, decisive, than visually observed, that could reduce the intensity of the referee work, can give a strong scientific basis for the athletes questioned the evaluation results.

\section{Game Environment Monitoring Sensors}

Windy impact on the track and field competition, when the wind speed exceeds the limit on the track and field venue, the created results will not be recognized. Outdoor race of the regatta, rowing competitions are needed anemometer to measure wind speed.

According to the working principle, anemometer has a wide variety in the form.

Using ultrasonic time or frequency difference method to achieve a speed of measurement is the revolution of mechanical wind instruments. Ultrasonic wave propagation speed in air and direction of airflow velocity stack. If the same with wind direction, its speed can be accelerated, conversely, speed slowly. Therefore, at a fixed detection conditions, ultrasonic wave propagating speed in air corresponds to a function of wind speed. It can calculate the accurate wind speed and direction.

As a result of acoustic wave propagation in the air, its speed is affected by temperature, but the anemometer is detection transmitting, receiving two channels on the two opposite direction, so the temperature on sonic velocity impact is negligible.

Ultrasonic anemometer can used in wind rapidly changing circumstances, and accurately measure wind speed average.

Because using advanced transducer as signal collecting device in the referee process, it greatly improves the accuracy of measuring results, utmost ground eliminates the artificial way of measuring the shortcomings to ensure that the game process and result fairness and authority.

\section{EXERCISE TRAINING DEVICE WITH SENSOR}

Modern sports are the epitome of strength and beauty, the pursuit of the goal is higher, farther, fast. Therefore, athletes usually use of various training equipment to improve the performance.

\section{A Weight Training Equipment Used in the Sensor}

The traditional weight equipment often only test one or two index of training, coaches can only understand roughly training intensity due to the weight and movement frequency. Born barbell weight and the impact force on the ground undoubtedly have a negative impact on psychology and the safety for athletes.

Along with the science and technology penetration, researchers have developed in the existing weight training equipment with displacement, speed, weight sensor, developed weight training data real-time acquisition and diagnostic analysis system to monitor the training process, strength, speed, power and other parameters, and to feedback the results of real-time to the user, thus realizing the eight training the intelligent and visualization and the safety of training.

\section{B Sensor Used in Taekwondo Simulation Training Instrument}

Athletes hit fast each other to achieve victory purpose mainly through walking mobile in Taekwondo training. 
Therefore, the force sensor becomes one of the main means of detection in existing Taekwondo simulation training instrument.

\section{1) Force Transducer}

To test whether the hit to the effective parts of the other and the size of the forces, it mounted force sensor in the target human body model on the hitting parts, there are resistance strain gauge, piezoresistive, and piezoelectric sensor. Resistive pressure sensor treats resistance strain gauges as the sensitive element, according pressure transmitted strain gauge resistance to measured force.It can be measured static force, dynamic forces. Piezoelectric force sensor is according to piezoelectric crystal generating charge in the dynamic force, only applies to the dynamic force measurement and the wide frequency range. Piezoresistive sensor more and more used widely with its good sensitivity, high accuracy.

2) Flexible Pressure Array Sensor

Test platform posed by the pressure dot sensor is a device training and evaluation taekwondo basic footwork and agility in different directions. Better distributed force sensor is flexible pressure array sensor

Flexible pressure array sensors are resistive sensor, its working principle is basically the same as ordinary resistive sensor. Sensor is used a screen printing technology to put sensitive material in a matrix print to the two insulating substrate (polyester film), the sense point corresponding position is made of through-holes to form the flexible force array and pressure array.

No force, the sensitive points of the upper and lower levels are not in contact, resistance was infinite. When strong plus in the sensor array, the upper and lower layers of the sensitive point conduction to format the contact resistance. The contact resistance decreases with the increase of the force, within a certain range, the variation is inversely proportional. The collected pressure signal put into the computer through the processing circuit for analysis in order to achieve the purpose of guiding training.

All hitting type athletic events, such as the martial arts, boxing, fencing, etc., can be applied to similar training equipment.

\section{IV.OTHER SPORTS MONITORING}

\section{A Movement Physiological Detection Sensor}

In the high-intensity training projects, detection athletes bone fatigue has important significance to improve the athlete's physique and protect the athlete's movement security, Nondestructive detection is a method using the ultrasonic Lamb wave propagating in the bone. Bone Young's modulus is as the measurement object. When bone fatigue, Young's modulus decreased to change the dispersion curve. Using the phase velocity to solve reversely the Young's modulus to know bone fatigue in order to achieve accurate non-destructive testing.

\section{B Fitness Equipment Sensor}

At the same time of promoting fitness exercise, a variety of fitness equipment is also vigorously developed.
Small equipment such as electronic grip force device is used strain gauge sensors and digital circuit to show the results of the forearm and hand muscles of strength; large fitness equipment such as intelligent running machine is used more types sensor: there are speed mileage, weight, heart rate sensor, so that the test results are displayed on a motorized treadmill, sports exercising can be well aware of the amount of exercise, physical condition. These additional fitness equipment for the scientific and technological element are more interesting and scientific than traditional equipment, loved by sports enthusiasts.

\section{Sadiums Construction Sensor}

Using sensing technology of the photovoltaic effect, solar panels is installed on the roof of the training hall of the National Stadium, the competition hall roof and south of the glass curtain wall. It is essentially a large area of the PN junction, when the sun lights to the PN junction, if the photon energy is larger than the band gap of the semiconductor material, a pair of free electrons and holes will produce rapidly diffusing from the surface inward, under the junction electric field, and finally establish the electromotive force of a light intensity, and thus, the light energy transmit into electrical energy for indoor power. Solar energy is clean, safe and reliable future energy, Sensor technology contributed for solar energy into electrical energy,

In Water Cube, temperature sensor applications in the pool, the detection of wall pressure parameters, water level control, induction flush valve, water meter ..

These examples are all used in sensor technology.

\section{V .SENSOR RESEARCH AND DEVELOPMENT TRENDS IN THE FIELD OF SPORTS}

Due to the important role of sensor in the information monitoring and control system, the countries of the world are very seriously its theoretical research and application of new materials, product development. Using the new science and technology in sports games can ensure the fair and equitable, such as a ball game, if having the electronic referee to judge the ball over the line; it will greatly reduce the controversy of judgment.

Optical fiber sensor is being universal attention because of its strong anti-interference, high sensitivity, light weight, small size, suitable for telemetry features. Currently, more than a number of mature products, such as optical torque sensor, as well as temperature, vibration, pressure sensor.

At the same time of the development and utilization of new materials, due to the development of microelectronics and micro-machining technology, the sensor is moving miniaturization, multi-functional, intelligent. Miniaturized sensors integrated package a single chip. Using micro-machining technology on the micron level sensing element, signal conditioning, data processing device. Because of its small size, the cheap price, easy integration, it can improve the accuracy of system testing, such as integrating miniature pressure sensors and micro-temperature sensor simultaneously measuring pressure and temperature, There are a lot of micro-sensors 
are available, such as pressure sensors, accelerometers. The multifunctional characteristics make the sensor can simultaneously detect two or more than two the characteristic parameter.

Because measured subjects of sporting events in motion, so much GPS and wireless sensor networks are free room for the development.

In addition, it is also important research topic about response time, electromagnetic interference, output and computer interface and other issues. With the development of electronic technology, sensor technology will certainly tend to improve. Applications will become more and more widely in the field of sports.

\section{REFERENCES}

[1]ZANG Yu-yang, YANG Di, GUO Xia-sheng, Study on Nondestructive Evaluation of Sports Bone Fatigue, J. China Sport Science and Technology Vol.44, No1, 2008, pp.40-42

[2] XIAO Yi, LIU Yu, Research on Plyometric Weight Training System Based on Displacement Transducer, J. Chine Sport Science Vol.29, No.4, 2009, pp,84-88

[3]HUANG Bao-hong, WANG Wei-xing, YANG Xian-jun. The Design and Application of the Agility Training Machine of Taekwondo Footwork [J]. China Sport Science and Technology Vol.45, No.2, 2009, pp, 57-60

[4] JIA Bo-nian, YU Pu, SONG Ai-guo, Sensor Technology, third ed., Southeast University Press , NanJing , 2007

[5] LIN Yu-chi, ZENG Zhou-mo, Modern sensor technology and system[J], China Machine Press, BeiJing,2009

[6] WANG Le-jun, GONG Ming-xin, HUANG Yong1,XU Yi-cheng, Research and Development of Multi-Parameter Synchronizing System in Sport Biomechanics[J].China Sport Science and Technology Vol.45,No.4,125-126,2009.

[7]GAO Zhi-hong, FENG Ju-tao, REN Wen-gang, QIN Zhi-ming, The Effects of New Rule and Electronic Protective Gear Application on Taekwondo Technique[J].China Sport Science and Technology Vol.46,No.4,86-89,2010.

[8] Howard J A, Valerie S H. Effects of Sterilization on the Tekscan Digital Pressure Sensor[J].Medical Engineering

Physics, 2003, pp, 775-780

[9]Ding T H, Chen X L, Huang Y P. Ultra-thin flexible eddy current sensor array for gap Measurements [J]. Tsing-hua Science \& Technology, 2004,9(6), pp,667-671

[10]Gray B L.Fearing R S.A Surface Micromachined Microtactile Sensor Array[J], Proc.of IEEE ICRA,1996,1-6 